\title{
Role of Combinatorial, Medicinal \& Biological Chemistry in Drug Discovery Development: An Introduction
}

\author{
Prakash Prajapat* \\ Department of Chemistry, Ganpat University, India
}

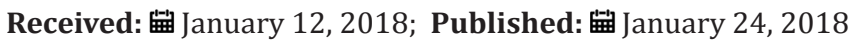

*Corresponding author: Prakash Prajapat, Department of Chemistry, Mehsana Urban Institute of Sciences, Ganpat University, Mehesana-384012, Gujarat, India

\section{Opinion}



Figure 1: Example of Combinatorial Chemistry.

Combinatorial chemistry, a new approach for greatly reducing the time for the discovery of newer organic motifs, has been developed over the last few years. Combinatorial chemistry (Figure 1) is a technique for creating huge numbers of biologically potent motif and their hybrids, then rapidly screening them for desirable properties 1. Almost pharmaceutical and agrochemical industries uses this strategy because of the potential for immense reducing in time and cost, and several drug candidates presently in clinical trials are products of this methodology. Drug discovery process (Figure $2 \& 3$ ) is long, time and money intensive process [1]. This science of has found application in the production of molecules of commercial interest in the construction of newer pharmacological active therapeutic agents derived from rational drug design, into synthesize complex natural molecules, in the finding innovative approaches to render this chemical science more efficient [2,3]. The development of newer pharmaceuticals is currently a critical and challenging task to the pharmaceutical industry. The vital interest of the medicinal and agrochemical industries in organic synthesis is often related with their natural occurrence. Similarly, medicinal and pharmaceutical field, there has always been and continue to be a need for newer chemical entities with diverse pharmacophoric properties. Many works are still needed to minimize the time, expenditure, and attrition rate in the drug discovery process simultaneously addressing the huge unmet medical need across the world.

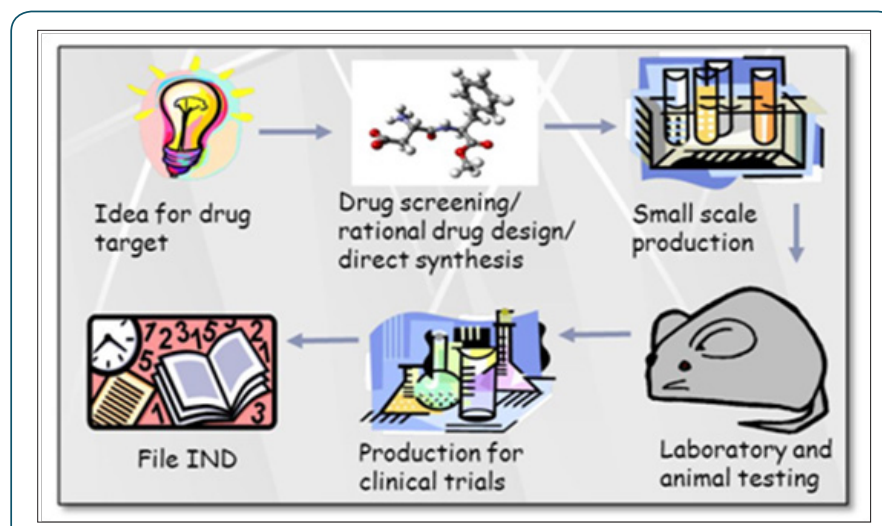

Figure 2: Drug development cycle.



Referencing the study report, poor pharmacokinetic and preclinical toxicity were the main reasons for the failure in the 
drug development, in addition to the lack of efficiency and adverse effects $[4,5]$. New drugs are necessitated to cure new diseases, to find less hazardous drug and to cure diseases whose drugs have become ineffective due to resistant strains of microorganisms. Besides these causes, new drug discovery and researches are required to recognize pharmacophore present in the effective drugs [6-8]. We must always continue to search for drugs which exhibit clear advantages over the already existing respective drugs. Such advantages may be improvement in bioactivity, partial or total absence of adverse effects, minor toxicity, more nutritive value, improved stability and decrease in production cost. In present, research development department (R\&D) of many pharmaceutical laboratories are working for synthesis of newer entities having improved drug activity and increasing yields of existing drugs [9-11]. Combinatorial, medicinal and biological chemistry have contributed to life processes and to the efforts to advance the quality of life as well as to the development of society from synthetic, biopharmaceutical, medical and industrial point of view. Finally, this communication opens the door to the combinatorial design, synthesis of biological active of a variety of newer hybrid molecules.

\section{Acknowledgement}

I thankful to chemistry staff members of the Mehesana Urban Institute of Sciences, Ganpat University, Gujarat for support..

\section{References}

1. Prajapat P, Talesara GL (2016) Synthesis and Anti-inflammatory Screening of Some Mono and Bis-Alkoxyphthalimide Linked Benzimidazole and their Quinazoline and Pyrimidine Derivatives. J Heterocyclic Chem 53(5): 163-1610.
2. Prajapat P, Vaghani H, Agarwal S, Talesara GL (2017) Synthetic and Medicinal Chemistry in Drug Discovery: Needs for Today. Ann Med Chem Res 3(1): 1021.

3. Prakash P (2018) Importance of Benzothiazole Motif in Modern Drug Discovery: Introduction. Mod Appro Drug Des 1(4): MADD 000517.

4. Prajapat P, Agarwal S, Talesara GL (2017) Significance of Computer Aided Drug Design and 3D QSAR in Modern Drug Discovery. J Med Org Chem 1(1): 1.

5. Prajapat P (2017) Utility of Drug Discovery in Medicinal and Organic Chemistry. Mod Chem Appl 5(4): 123.

6. Prajapat P, Kumawat M, Kherodiya B, Talesara GL (2016) An expedient synthesis and antimicrobial evaluation of ethoxyphthalimido derivatives of pyrimido[4,5-e]pyrimidine analogues from 1-(1H-benzimidazol-2-yl) guanidine. J Indian Chem Soc 93: 539-544.

7. Prajapat P, Rathore KK, Hussain N, Yogi PP, Talesara GL (2015) Synthesis of novel pyrimidines, pyrimidopyrimidines and their oxygen substituted hydroxylamine derivatives as potential pharmacological interest. Iranian Journal of Organic Chemistry 7(3): 1605-1612.

8. Agarwal S, Kalal P, Gandhi D, Prajapat P (2017) Thiazole containing Heterocycles with CNS activity. Curr Drug Discov Technol 14.

9. Kumawat M, Kherodiya B, Prajapat P, Talesara GL (2015) Synthesis of alkoxyphthalimide derivatized oxoimidazolidinyl oxazolo/thiazolo dihydropyrimidine and oxoimidazolidinyl tetrahydropyrimidine via common Schiff base intermediate and evaluation of their antibacterial activity. Indian J Chem 54:117-127.

10. Kherodiya B, Prajapat P, Kumawat M, Talesara GL (2015) Synthesis and antimicrobial evaluation of bis imidazolidinone assembled dihydropyridine ethoxyphthalimide derivatives. Iranian Journal of Organic Chemistry 7(4): 1661-1668.

11. Prajapat P, Rathore KK, Gandhi D, Agarwal S, Hussain N, et al. (2016) A Facile Synthesis of Biologically Significant 2-(1,3-benzothiazol2-ylimino)-1,3-thiazolidin-4-one/3-(1,3-benzothiazol-2-yl)-2thioxoimidazolidin-4-on Analogues from 1-(1,3-benzothiazol-2-yl) thiourea and their Alphahydroxylamine Derivatives. Iranian Journal of Organic Chemistry 8(2): 1795-1801.

\section{(C) (P) This work is licensed under Creative}

Submission Link:

Submit Article

DOI: 10.32474/AOICS.2018.01.000104



